\title{
Integrating a Facially Driven Treatment Planning to the Digital Workflow for Rehabilitation of Edentulous Arches: A Case Report
}

\author{
Jesus Creagh ${ }^{1}$, Lauren Bohner ${ }^{2}$, Newton Sesma ${ }^{3}$, Christian Coachman ${ }^{4}$
}

\begin{abstract}
Aim and objective: This case report presents the integration of a digital facially driven prosthetic plan to the computer-assisted implant planning for rehabilitation of edentulous arches.

Background: Diagnosis of edentulous arches is hampered by the lack of intraoral references. However, a digital facial profile analysis facilitates the treatment plan, taking into consideration the harmony among teeth, lips, and face to restore a pleasant smile.

Case description: The first appointment consisted of digital documentation including intraoral scans, facial scans, and photographs. Based on these data, a smile frame was created to guide the digital wax-up. Cone-beam computed tomography (CBCT) scans were merged to facial and intraoral scans to perform the virtual surgical planning. Integration between facial, intraoral, and bone tissues were used as a reference to define implant position and prosthetic planning. The digital planning was integrated into the surgical procedure using stackable templates, and an immediate loading was performed. The interim prosthesis was manufactured based on the digital wax-up. With digital data, quality control could be performed to evaluate the esthetic outcome of the treatment.

Conclusion: An esthetic and functional rehabilitation was possible using the respective digital workflow to define harmony between a smile and facial tissues.

Clinical significance: A digital treatment implant planning can be conducted considering patient's individual needs to improve the esthetic outcome.

Keywords: Computer-aided design, Computer-aided manufacturing, Computer-assisted, Dental implants, Surgery.

The Journal of Contemporary Dental Practice (2020): 10.5005/jp-journals-10024-2985
\end{abstract}

\section{INTRODUCTION}

Extensive rehabilitation with fixed implant-supported prosthesis requires the development of a careful treatment plan taking into consideration the relation between hard and soft tissues. As a diagnosis of edentulous arches is particularly hampered by the lack of intraoral references, facial profile analysis plays an important role in tooth arrangement. ${ }^{1-3}$ The more the clinician restores the harmony among teeth, lips, and face, the better the esthetic outcome. $^{4}$

The advance of digital technologies has facilitated the prosthetic planning of edentulous patients. By creating a virtual patient, it is possible to understand the patient's needs and visualize the esthetic effect of the treatment. Thus, instead of following fixed paradigms to define the smile line and teeth shape, treatment can be performed in accordance with the patient's individual features. $^{4-7}$

However, the use of computer-aided design (CAD) technology to obtain functional and esthetic records of edentulous patients is still challenging. Therefore, additional resources may be useful for determining the ideal tooth arrangement. For instance, a smile frame can be designed to guide a digital facially driven treatment planning.

Regarding implant therapy, a challenge of CAD-CAM (computer-aided design, computer-aided manufacturing) dentures is to transfer the information acquired digitally to the clinical field. Mucosa-supported guides are used as a template to define the implant position according to the digital planning. However, its stability depends on the mucosa resilience and requires the use

\footnotetext{
${ }^{1}$ Universidad Católica San Antonio (UCAM), Sevilla, Spain

${ }^{2}$ Department of Oral and Maxillofacial Surgery, Hospital University Münster, Münster, Germany

${ }^{3}$ Department of Prosthodontics, University of São Paulo, São Paulo, Brazil

${ }^{4}$ Department of Restorative Dentistry, University of Pennsylvania, Philadelphia, USA; DSD Founder, São Paulo, Brazil
}

Corresponding Author: Lauren Bohner, Department of Oral and Maxillofacial Surgery, Hospital University Münster, Münster, Germany, Phone: +49 252 83-47013, e-mail: lauren.bohner@ukmuenster.de

How to cite this article: Creagh J, Bohner L, Sesma N, et al. Integrating a Facially Driven Treatment Planning to the Digital Workflow for Rehabilitation of Edentulous Arches: A Case Report. J Contemp Dent Pract 2020;21(12):1393-1397.

Source of support: Nil

Conflict of interest: Coachman C is co-founder of DSD Digital Smile Design.

of anchor guides. In this respect, an inaccurate support guide can lead to potential errors. ${ }^{8}$

The present case report describes the rehabilitation of edentulous arches by a full digital workflow. Digital profile analysis was added to the computer-assisted implant planning to ensure the esthetic outcome. To ensure surgical accuracy, stackable guides were used to guide the surgery. By using this approach, a minimally invasive treatment was conducted to restore function and esthetics with a CAD-CAM immediate prosthesis. 

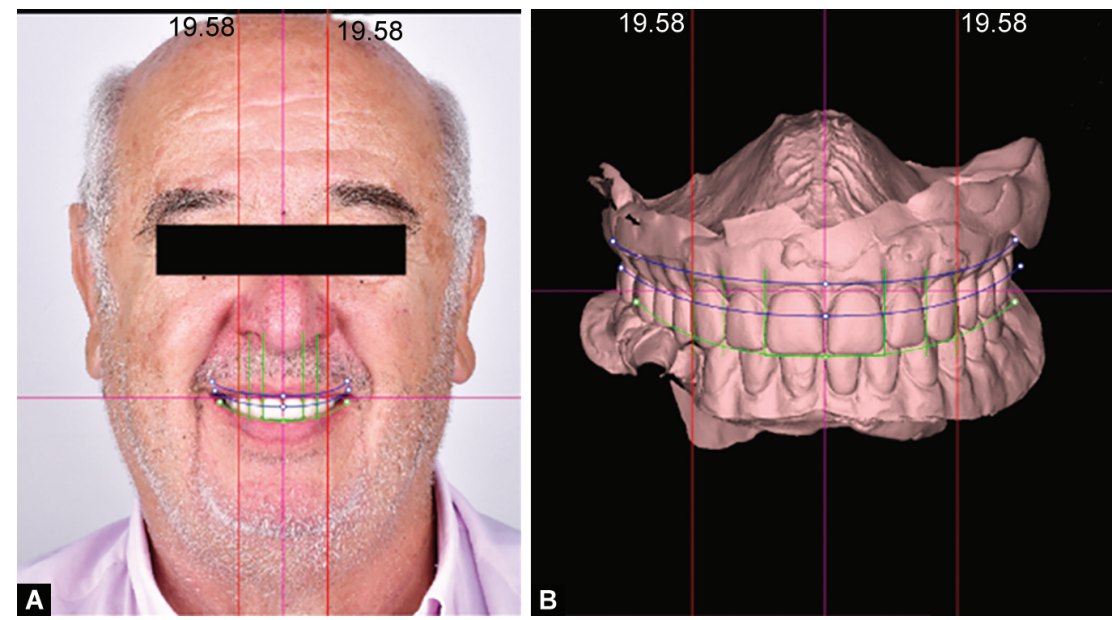

Figs $1 \mathrm{~A}$ and B: Digital smile design protocol. Treatment planning is based on facial reference lines and following esthetic principles: (A) Facial analysis; (B) Digital smile design
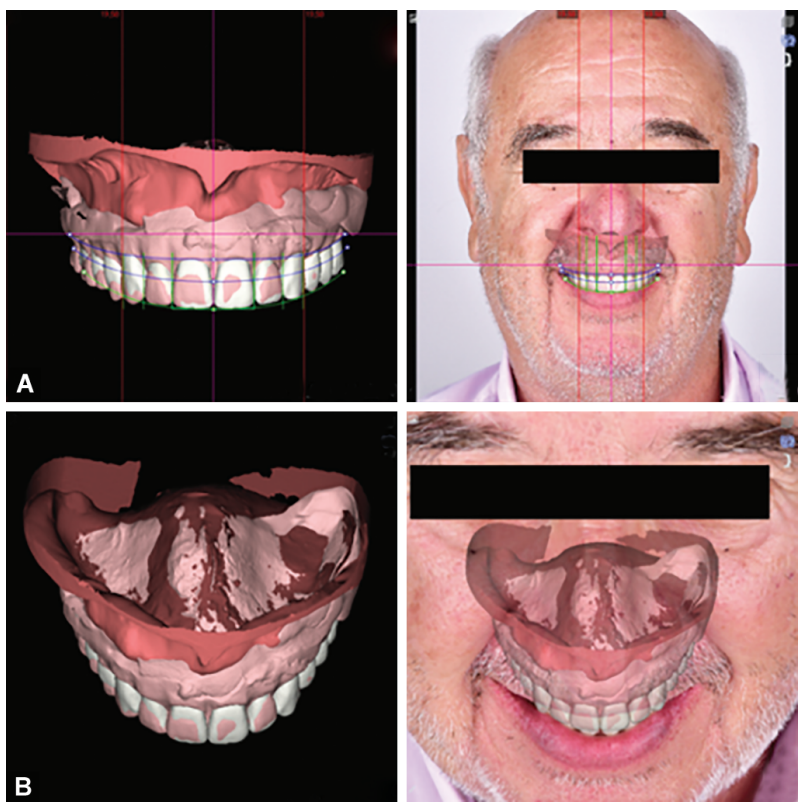

Figs $2 \mathrm{~A}$ and B: Digital wax-up. The association between photographs and intraoral scans helps to visualize the harmony between a smile and facial profile. Files can be visualized from different angles: (A) Facial profile photo; (B) 12-o'clock photo

\section{Case Description}

A 71-year-old patient was referred to the practice for dental implant rehabilitation. Anamnesis, clinical and radiographic examination revealed maxillary and mandibular edentulous arches with the absence of abnormalities or pathologies. Conventional dental prostheses were functionally satisfactory but required adjustments to improve esthetics.

At the first consultation, photographs, videos, intraoral digital impression, and radiographs were taken to perform a facially guided rehabilitation. The treatment plan was performed with the Software Nemo Smile Design (Nemotec) and a digital smile design (DSD) protocol was applied. Using the conventional prostheses as a reference, a bidimensional smile frame was drawn over the facial photograph of the patient. This smile frame represents the ideal smile, on which the teeth position and shape are determined based on facial reference lines and following esthetic principles.

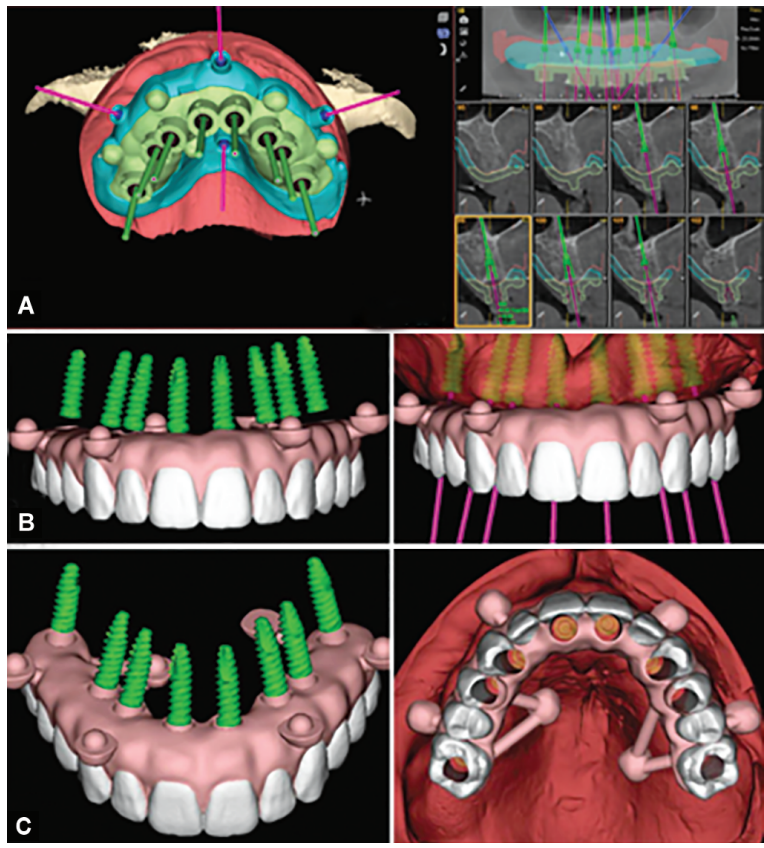

Figs $3 \mathrm{~A}$ to C: Dental implant surgical planning. The integration between intraoral and CBCT scans allows determining the implant position taking into consideration prosthetic needs and anatomical structures

The smile frame and the digital three-dimensional (3D) model were then overlapped to create the digital wax-up design (Fig. 1). To verify the harmony between the designed smile and the face, as well as functional principles, the digital wax-up was superimposed onto facial scans and photos taken in different angulations. Facial harmony, vertical dimension, occlusion, and intermaxillary relationship were evaluated digitally (Fig. 2).

After the patient's approval, a surgical plan was conducted. In this step, a cone-beam computed tomography (CBCT) was taken to assess the relation between soft and hard tissues. Computerassisted virtual surgical planning included the insertion of eight and six implant insertions in maxillary and mandibular areas, respectively.

The superimposition of CBCT data and intraoral scans allowed us to determine the ideal implant position in accordance with anatomical structures and prosthetic design (Fig. 3). Stackable 
surgical guides were designed digitally and printed to orient implant perforations. In this case, three surgical guides and a provisional restoration were printed (Fig. 4). Three stackable surgical guides and a provisional restoration were designed digitally and printed to orient implant placement.

Maxillary and mandibular implant surgeries were conducted using a flapless approach. After local anesthesia, the base guide was positioned at the mucosa and fixed by anchor guide pins. This guide aimed to stabilize further guides and remained fixed to the mucosa during the surgical procedure. Implants insertion was performed using an implant guide. Eight dental implants (Bioner System) and six dental implants (Top DM, Bioner System) were placed in maxilla and mandible, respectively. Implant position was verified using a multifunctional guide. Immediate provisionalization and loading were possible due to the appropriate insertion torque. Abutments were fixed to the dental implants and the immediate provisional restoration was attached to the base guide. Provisional titanium cylinders were connected to the restoration with acrylic resin. Then, the base guide was removed and a CAD-CAM interim prosthesis was screwed after ensuring appropriate occlusion (Fig. 5). Figure 6 shows the final results with the return of function and esthetics.

\section{Discussion}

Extensive rehabilitation of edentulous arches is challenging since these are associated with a compromised esthetic and occlusal deficiencies. Usually, visual perception of facial harmony is hampered by the lack of adequate anatomical references. Conventionally, teeth position is guided by the information established on occlusal rims. ${ }^{9,10}$

Currently, digital technologies offer the possibility to simulate the expected outcome in virtual patients. Thus, the treatment plan can be driven according to the patient's features. This concept can be used for different prosthetic solutions and has acquired special importance for dentures. ${ }^{11-14}$

Lo Russo et al. ${ }^{5}$ described a digital workflow for tooth arrangement using facial and intraoral scans. However, scans were not able to provide dynamic movements, and the maxillomandibular relationship must be conducted manually based on the occlusal rims. In our case report, a digital facial analysis improved the harmony between facial and intraoral tissues. Thus, tooth arrangement was facilitated using the planned restoration as a reference for the fabrication of provisional and definitive restorations.

The process of creating a smile frame and conducting a facial analysis was facilitated by an adequate old prosthesis, which was

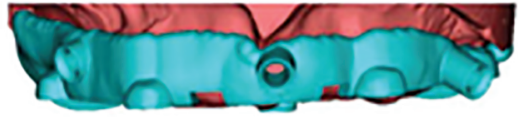

2. base gide with pins + implant guide

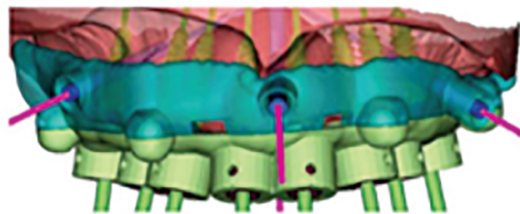

3. base gide with pins + multifunctional guide
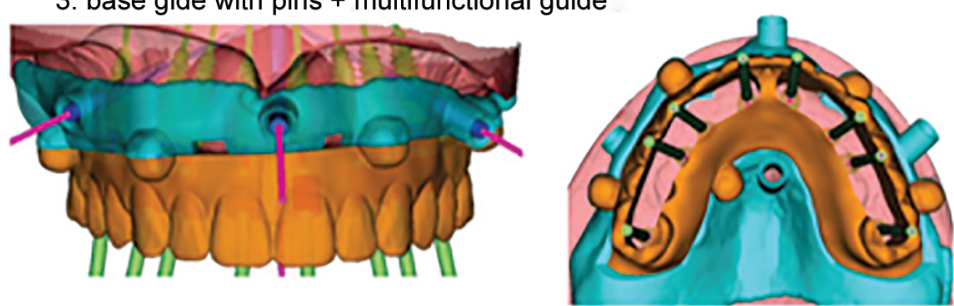

4. base gide with pins + hybrid provisional with pink gum
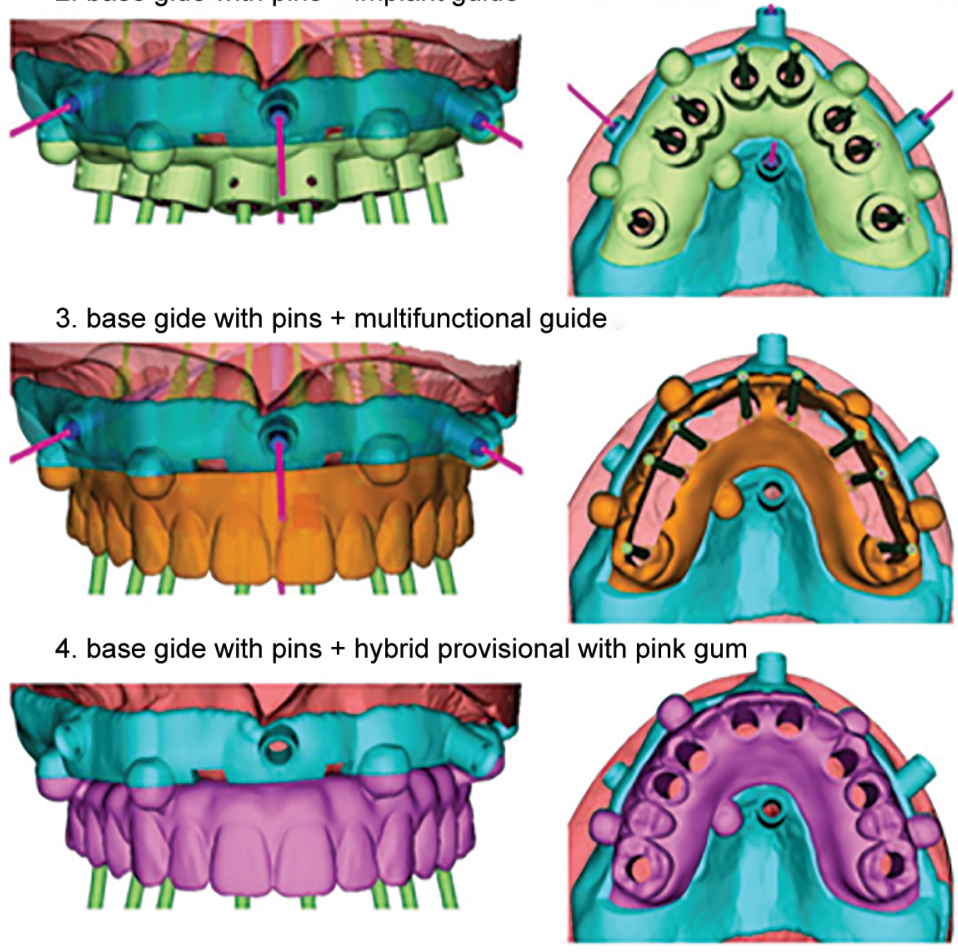

1. base gide
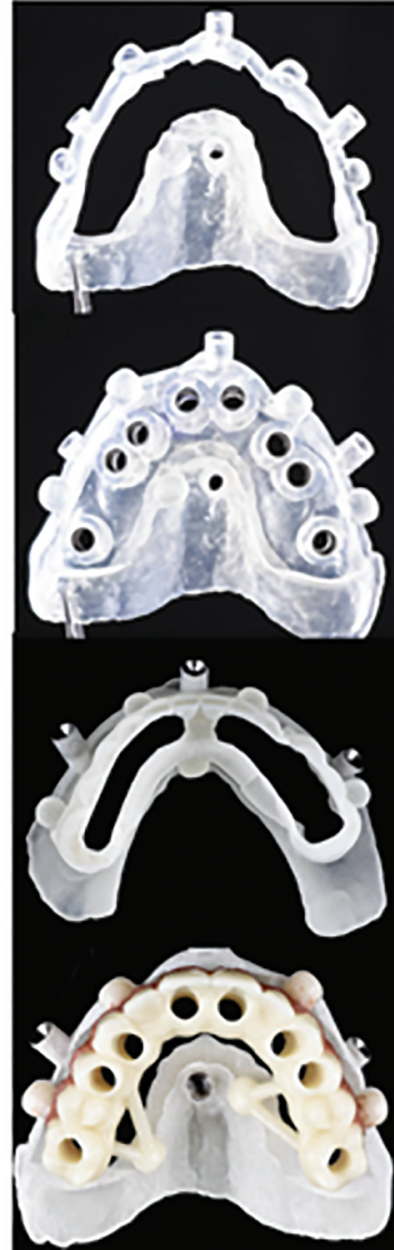

Fig. 4: Three surgical guides and a provisional restoration were printed: (1) Base guide is anchored to the mucosa using pins. (2) The implant guide is adapted to the base guide to guide the implant perforations. (3) A multifunctional guide allows evaluating the positioning of dental implants according to the prosthetic plan. (4) A provisional restoration can be adjusted for immediate loading. The adaptation of prostheses to the base guide ensures the correct placement, determined during the digital planning 

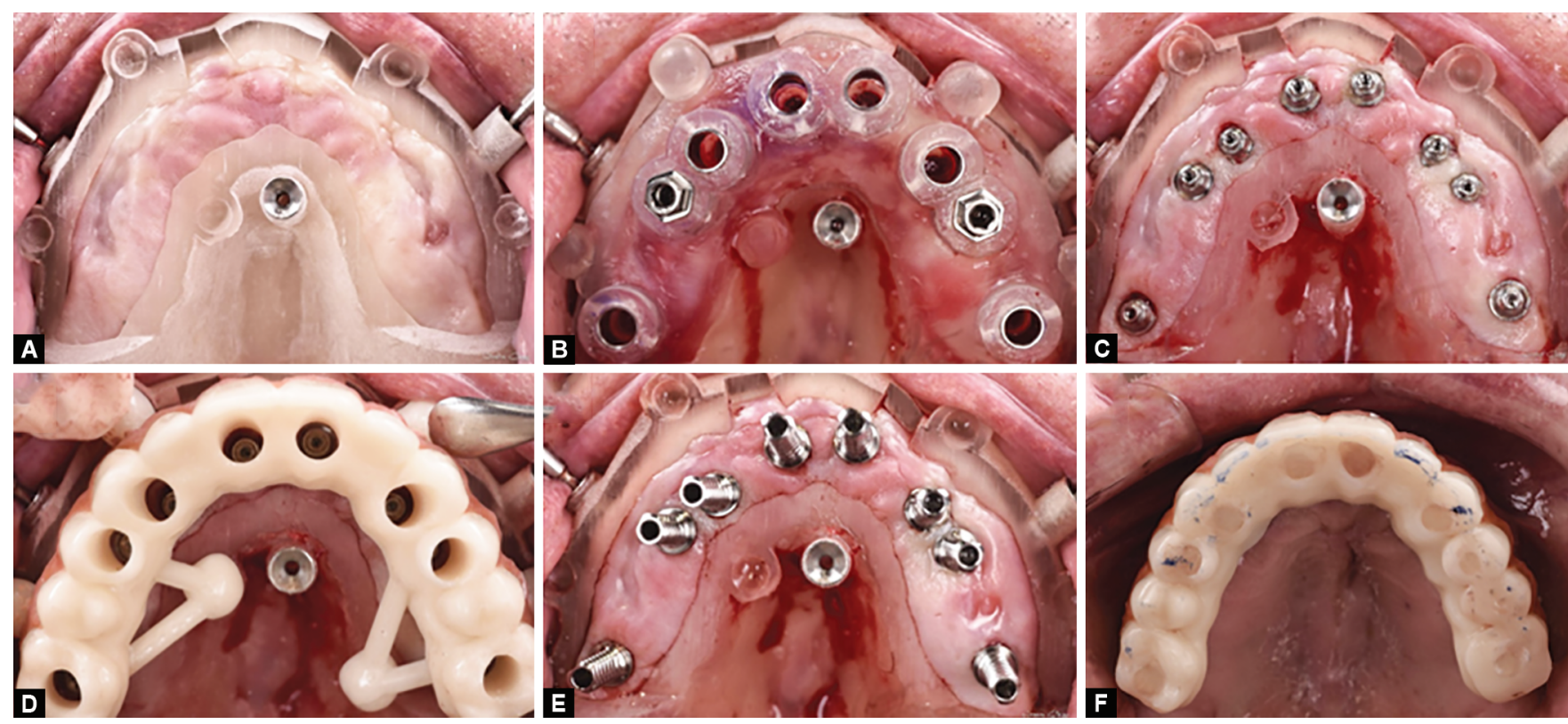

Figs 5A to F: Surgical guides showed clinically. From left to right: (A) Base guide was positioned at the mucosa and fixed by anchor guide pins (B) Implant perforations were guided by an implant guide; (C and D) Abutments were fixed to the dental implants and the immediate provisional restoration was attached to the base guide; (E) Provisional titanium cylinders were connected to the restoration with acrylic resin; (F) Installation of a CAD-CAM interim prostheses
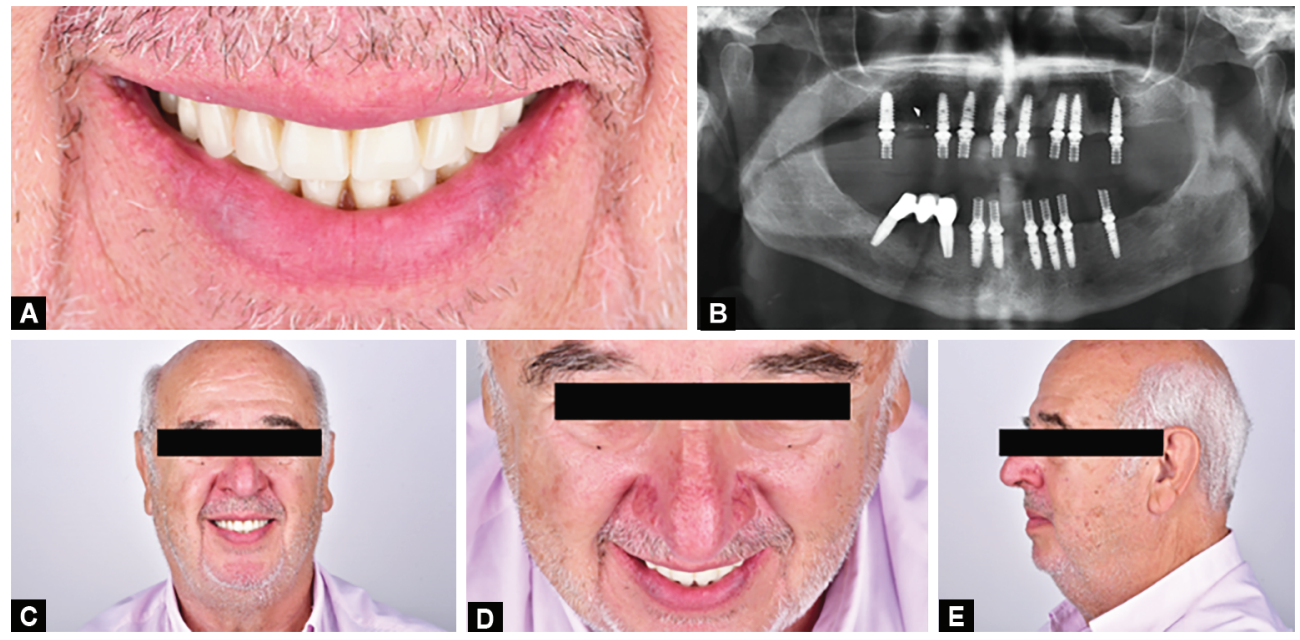

Figs 6 A to E: Immediate loaded interim prostheses

used as a reference to the future restoration. Digital documentation of a patient's smile enabled us to overcome the limitations of the old restoration and to design a new smile based on functional and esthetical principles.

Furthermore, different anatomical structures, as face, bone, and teeth were integrated, allowing to visualize changes before and after the treatment. ${ }^{15}$ Treatment simulation can also be used as a motivation tool to explain and show the patient the treatment steps. For this purpose, a motivational mock-up is a valuable tool to bring the digital plan to the clinical situation. As soon as the patient approves the planned result, different treatment options can be offered. ${ }^{6}$ In this case report, treatment costs and fragile prognosis influenced the patient to undergo the implant therapy.

The digital planning and the use of stackable support-guides allowed a minimally invasive approach. Flapless surgeries provide greater patient comfort and reduce surgical time and postoperative bleeding. This is especially beneficial for patients with systemic diseases, who might develop complications due to invasive surgical approaches. $^{16}$

Despite these benefits, the lack of stabilization of mucosa supported guides during the surgical procedure can lead to inaccuracy and result in inadequate implant placement. In this condition, dental implants could be placed in a different position than originally planned. By using stackable surgical guides, the implant position is determined taking into consideration prosthetic needs and anatomical structures. The digital plan can be reproduced in a surgical procedure to ensure proper prosthesis installation as simulated digitally. In this case report, three surgical guides were used to guarantee a proper implant position.

After 6 months, quality control was conducted digitally. By overlapping intraoral scans of restorations, it is possible to control 
functional and esthetic parameters and to compare them with previous scans or with the planned restoration. Thus, acrylic wear, occlusion changes, or the need for adjustments can be easily visualized.

\section{CONCLUSION}

An optimal esthetic and functional rehabilitation was acquired with the described digital workflow. The integration of a facially driven treatment planning to the computer-assisted surgery improved the esthetical outcome, and the use of stackable guides allowed a minimally invasive approach ensuring surgical accuracy. However, the conductance of a full digital workflow was only possible because the old prosthesis was considered functionally satisfactory. This protocol is recommended for cases in which the main purpose is to restore or to improve the esthetical outcome.

\section{ACKNOWLedgments}

The authors would like to thank the technician Guglielmo Parziale (Napoles, Italy) for his amazing work in manufacturing the prosthesis showed in this case.

\section{References}

1. Mericske-Stern RD, Taylor TD, Belser U. Management of the edentulous patient. Clin Oral Implants Res 2000;11:108-125. DOI: 10.1034/j.1600-0501.2000.011S1108.x.

2. Lee DJ, Saponaro PC. Management of edentulous patients. Dent Clin North Am 2019;63(2):249-261. DOI: 10.1016/j.cden.2018. 11.006 .

3. Coachman C, Calamita MA, Coachman FG, et al. Facially generated and cephalometric guided 3D digital design for complete mouth implant rehabilitation: a clinical report. J Prosthet Dent 2017;117(5):577-586. DOI: 10.1016/j.prosdent.2016.09.005.

4. Mahn E, Sampaio CS, Pereira da Silva B, et al. Comparing the use of static versus dynamic images to evaluate a smile. J Prosthet Dent 2020;123(5):739-746. DOI: 10.1016/j.prosdent.2019.02.023.
5. Lo Russo L, Di Gioia C, Salamini A, et al. Integrating intraoral, perioral and facial scans into the design of digital dentures. J Prosthet Dent 2020;123(4):584-588. DOI: 10.1016/j.prosdent.2019.05.030.

6. Coachman C, Georg R, Bohner L, et al. Chairside 3D digital design and trial restoration workflow. J Prosthet Dent 2020(5):In Press 10.1016/j. prosdent.2019.10.015.

7. Li J, Sommer C, Wang HL, et al. Creating a virtual patient for completely edentulous computer-aided implant surgery: a dental technique. J Prosthet Dent 2020. In Press 10.1016/j.prosdent.2020.02.026.

8. Gallardo YNR, Silva-Olivio IRT, Mukai E, et al. Accuracy comparison of guided surgery for dental implants according to the tissue of support: a systematic review and meta-analysis. Clin Oral Implants Res 2017;28(5):602-612. DOI: 10.1111/clr.12841.

9. Lemos CAA, Verri FR, Gomes JML, et al. Bilateral balanced occlusion compared to other occlusal schemes in complete dentures: a systematic review. J Oral Rehabil 2018;45(4):344-354. DOI: 10.1111/ joor.12607.

10. Tang J, Wang J, Wang Z, et al. Facial aesthetic evaluation of rehabilitation effects in edentulous patients with varying degrees of residual ridge resorption by 3D stereophotogrammetry. J Oral Rehabil 2020(9):In Press 10.1111/joor.13035.

11. Cascón WP, Gopegui JR, Revilla-León M. Facially generated and additively manufactured baseplate and occlusion rim for treatment planning a complete-arch rehabilitation: a dental technique. J Prosthet Dent 2019;121(5):741-745. DOI: 10.1016/j. prosdent.2018.07.009.

12. Neumeier TT, Neumeier H, Peterson G. An easy transition from conventional to digital dentures for an edentulous patient: a case report. Gen Dent 2020;68:50-55.

13. Joda $\mathrm{T}$, Zarone F, Ferrari M. The complete digital workflow in fixed prosthodontics; a systematic review. BMC Oral Health 2017;17(1):124. DOI: 10.1186/s12903-017-0415-0.

14. Oh JH, An X, Jeong SM, et al. Digital worflow for computer-guided implant surgery in edentulous patients: a case report. J Oral Maxillofac Surg 2017;75(12):2541-2549. DOI: 10.1016/j.joms.2017.08.008.

15. Joda T, Gallucci GO. The virtual patient in dental medicine. Clin Oral Implants Res 2015;26(6):725-726. DOI: 10.1111/clr.12379.

16. Wismeijer D, Joda T, Flügge $T$, et al. Group 5 ITI consensus report: digital technologies. Clin Oral Implants Res 2018;29(S16):436-442. DOI: $10.1111 / \mathrm{clr} .13309$ 